\title{
Cryogenic ASICs in GaAs for Applications with Particle Detectors
}

\author{
D.V. Camin and G. Pessina \\ Dipartimento di Fisica dell'Università and INFN, Istituto Nazionale di Fisica Nucleare, Via Celoria 16, \\ 20133 Milano, Italy
}

\begin{abstract}
We present two cryogenic Application Specific Integrated circuits (ASIC's) realized with a GaAs MESFET process. A single-channel differential or double-channel single-ended voltage sensitive preamplifier for $4.2 \mathrm{~K}$ operation, to be used with bolometric detectors, was realized and tested. A very simple structure working as a unity gain buffer or as a transconductance amplifier, or even as a shaping filter was tested in view of an application with a fast particle detector operated at liquid Argon temperature.
\end{abstract}

\section{INTRODUCTION}

There are several experiments in elementary particle physics which use detectors operating at cryogenic temperatures. For optimum operation, these instruments need to be readout with amplifiers able to operate in the same environment, at low temperature.

Bolometers are very high energy resolution detectors [1] which operate at temperatures as low as 10 $\mathrm{mK}$. The energy deposited in the detector by an impinging particle suddenly increases the crystal temperature. The change of temperature is converted into an electrical signal by a suitable sensor put in good thermal contact with the detector absorber. The relaxation to the initial conditions is made through a thermal link which connects the system to the heat sink.

There are a few classes of such detectors, depending on their composition and dimensions. Large mass bolometric detectors consist of two main parts: the absorbing crystal and the temperature sensor. Monolithic bolometers are made with crystals, often silicon, of very small mass and the thermal sensor is directly implanted in a small region.

Various type of thermal sensors can be used to read the temperature change: Silicon or Germanium Thermistors, Superconducting Tunnel Junctions (STJ) or Transition Edge (TE) superconducting films.

Thermal detectors are slow, rise time being limited by the speed of sound in the absorbing crystal. The frequency bandwidth of the signals generated is limited to a few $\mathrm{kHz}$. The intrinsic energy resolution achievable can be very high, of the order of a few electron-volt. The impedance have a dominant resistive component ranging from a few $\Omega$ (TE sensor) to hundred of $M \Omega$ (Thermistors).

Frequently, a limit in achieving the intrinsic energy resolution arise from noise in the detector correlated to external disturbances. For instance, mechanical vibrations in the detector creates, by friction, spurious noise spikes and, in the case of thermistors sensors of large impedance, the vibration of the electrical link generates microphonic noise in the electrical parasitic capacitance shunting the sensor.

In this paper two monolithic amplifiers designed for applications with two different detectors and experimental conditions are presented. They have been realized using a GaAs MESFET process.

In Section 2 a Differential/Single-ended Voltage-Sensitive Preamplifier (D/SVSP) is described. It is intended for application with bolometric and Superconducting Tunnel Junction detectors.

In Section 3 a Buffer/Led Driver (B/LD) circuit for fast signal handling is presented. 


\section{THE DIFFERENTIAL/SINGLE-ENDED VOLTAGE SENSITIVE PREAMPLIFIER (D/SVSP)}

\subsection{Description of the circuit configuration}

For the above mentioned reasons the preamplifier circuit must have low series noise in the low frequency region and must be capable to operate at temperatures as close as possible to 1 Kelvin, to minimize the length of the link. Moreover, the microphonism originating in the electrical link can be strongly attenuated by adopting a preamplifier with differential input. This way a pair of wires firmly tied together, connecting the sensor to the preamplifier, generates only common mode disturbances. When the wires vibrates, those disturbances will cancel out at the preamplifier output. Nevertheless, with low resistive sensors the differential configuration may be not requested and a single-ended configuration would be preferred.

Having in mind the above requirements we have designed and realized a D/SVSP capable to operate as a differential voltage preamplifier or as a two channel single-ended voltage preamplifier.

Figure 1 shows the schematic of the D/SVSP. When operated as a single-ended preamplifier, two identical channels are available. The network of Figure 1 is symmetrical at the left and right of the two output pads OUT $^{+}$and OUT. For this operating mode pad $S_{1}$ must be connected to $V_{S s}$, while pad $S_{2}$ to

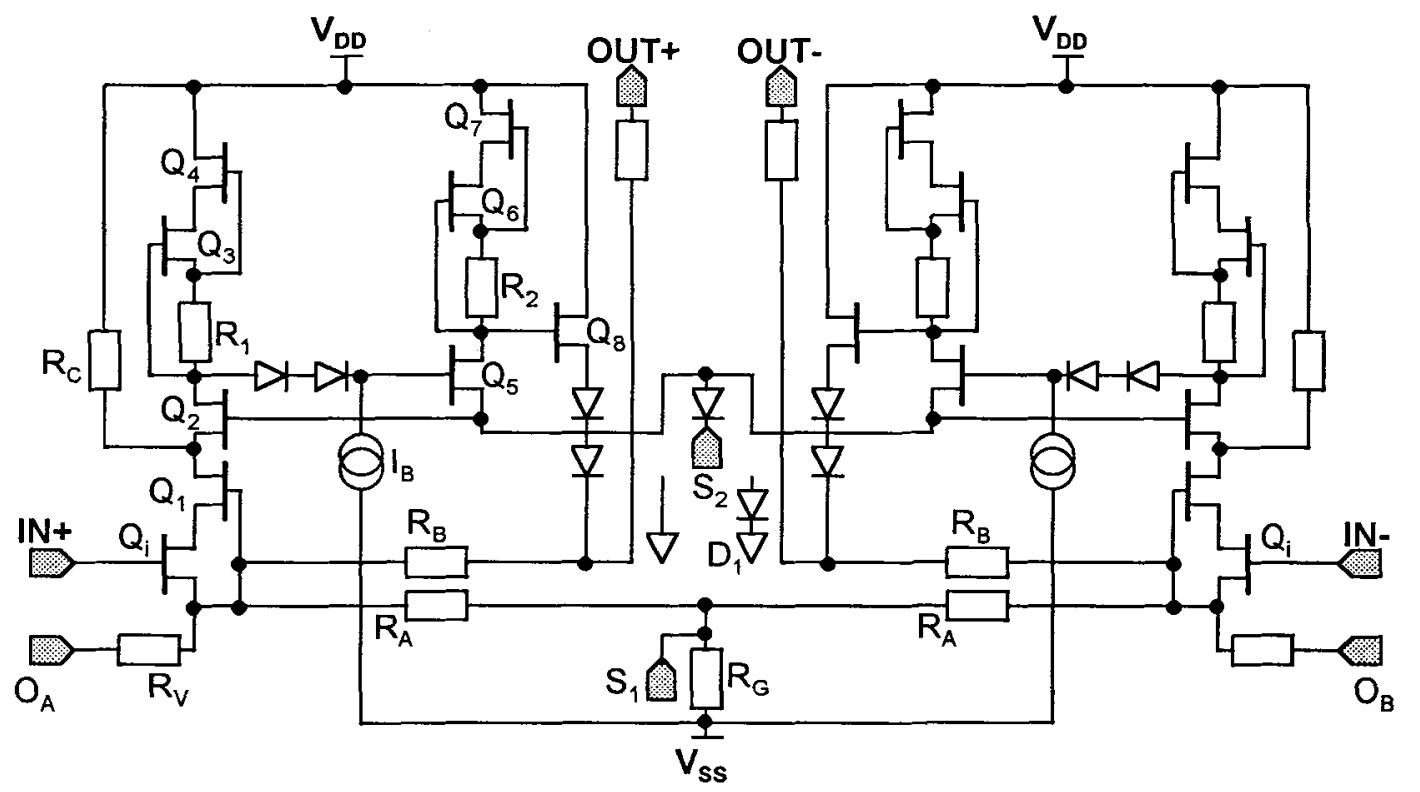

Figure 1: Schematic diagram of the D/SVSP.

diode $D_{1}$. Each channel features a closed loop gain of 11 which is given by $\left(R_{B} / R_{A}+1\right), R_{B}$ being $1 \mathrm{k} \Omega$, $R_{A} 100 \Omega$. High open-loop gain is assured by the dual stage configuration. $Q_{i}$ is the input transistor, $Q_{1}$ bootstraps it and $Q_{2}$ cascodes the structure. $R_{1}, Q_{3}$ and $Q_{4}$ form a bootstrapped current source which loads this input stage. The inverting stage is a common source amplifier, $\mathrm{Q}_{5}$, loaded with another bootstrapped current source $R_{2}, Q_{6}$ and $Q_{7}$. Transistor $Q_{8}$ buffers the output of the whole preamplifier. The two diodes at the drain of $Q_{2}$ and the current generator $I_{B}$ serve as level voltage translators. The input transistor $Q_{i}$ has an area $(\mathrm{L} \times \mathrm{W}) 3 \times 6000 \mu^{2}$ to assure low series noise. $\mathrm{A} \mathrm{L}=3 \mu \mathrm{m}$ gate-length was selected as the most suitable for low $1 / \mathrm{f}$ noise [2], [3]. The whole monolithic chip area is $2.5 \times 2.5 \mathrm{~mm}^{2}$. The resistor $R_{C}$ is used to inject a suitable biasing current, in the range $1.8 \mathrm{~mA}$ to $2.5 \mathrm{~mA}$, into $\mathrm{Q}_{i}$. The preamplifier was designed to get minimum power dissipation. The positive supply voltage $V_{D D}$ may be $4 \mathrm{~V}$ or $5 \mathrm{~V}$, depending on the noise and dynamic requirements. $V_{S S}$ for the single ended configuration is approximately 0.3 to $0.6 \mathrm{~V}$, 
adjustable to get proper DC output voltage, at half of the allowed output swing, which ranges from 1.4 to $2 \mathrm{~V}\left(\mathrm{~V}_{\mathrm{DD}}=3.5 \mathrm{~V}\right)$ or $3 \mathrm{~V}\left(\mathrm{~V}_{\mathrm{DD}}=4.5 \mathrm{~V}\right)$. Power dissipation is $25 \mathrm{~mW}$ and $32 \mathrm{~mW}$ for $\mathrm{V}_{\mathrm{DD}}$ equal to $4 \mathrm{~V}$ and $5 \mathrm{~V}$ respectively.

For differential operation pad $S_{1}$ has to be left open, pad $S_{2}$ must be connected to ground. In this way the resistor $R_{G}$ sinks the network current and the two stages become coupled at the sources of the two input transistors $Q_{i}$. The positive supply voltage $V_{D D}$ can range now from 3 to $4 \mathrm{~V}$, while the negative supply voltage $V_{S S}$ varies between -2.5 to $-3 \mathrm{~V}$, depending on the desidered output voltage operation. Power dissipation ranges from $21 \mathrm{~mW}$ to $37 \mathrm{~mW}$. The output differential voltage (OUT ${ }^{+}-$OUT $^{-}$) is now 11 times the input differential voltage $\left(\mathrm{IN}^{+}-\mathrm{IN}\right)$. The signal swing is the same as for the single ended configuration, but the output $\mathrm{DC}$ bias now stabilize between $0.6 \mathrm{~V}$ to $1 \mathrm{~V}\left(\mathrm{~V}_{\mathrm{DD}}=3 \mathrm{~V}\right)$ or $2 \mathrm{~V}\left(\mathrm{~V}_{\mathrm{DD}}=4 \mathrm{~V}\right)$, equivalent to the voltage across the missing diode.

The most relevant characteristic obtained with this design is that the very high input impedance differential preamplifier is similar to an instrumentation amplifier, but uses only one long-tailed pair at the input, saving a factor of $\sqrt{ } 2$ in the series noise.

For both configurations two additional pads are available, $\mathrm{O}_{\mathrm{A}}$ and $\mathrm{O}_{\mathrm{B}}$, which allow to equalize the output voltage at the same DC level. Alternatively, either one or both of them can be used to trim the output while maintaining fixed the negative supply $V_{S S}$ at a desidered value.

As can be observed by inspection the preamplifier is totally DC coupled.

\subsection{Results obtained}

Concerning the dynamic behavior, the D/SVSP showed very good results. The $1 / \mathrm{f}$ noise, instead, was not as low as expected according to noise performances of previous monolithic runs.

For the differential configuration when $\mathrm{V}_{\mathrm{DD}}$ is $3 \mathrm{~V}$ the integral non-linearity was less then $0.5 \%$ referred to a $300 \mathrm{mV}$ range, as can be seen in Figure 2 where the response to a $100 \mathrm{kHz}$ sinusoidal signal is shown. The common mode rejection ratio (CMRR) was also high, being about $75 \mathrm{~dB}$ from DC to a frequency of a few $\mathrm{kHz}$, remaining larger than $65 \mathrm{~dB}$ up to $100 \mathrm{kHZ}$, Figure 3 . For the single-ended configuration the same results applies except that CMRR should not be considered. The level of possible cross-talk between the two channels was very small, difficult to quantify by direct measurements.

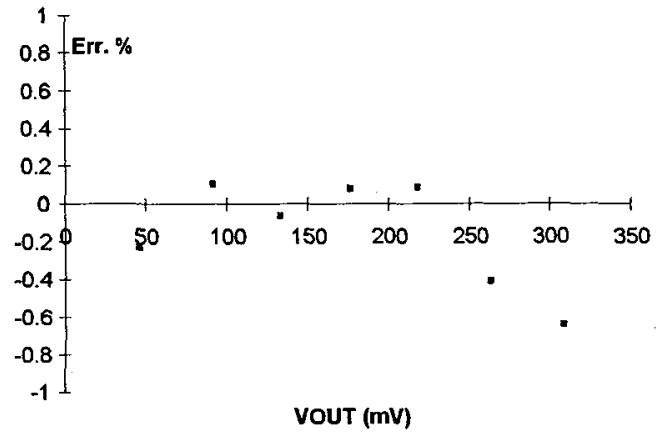

Figure 2: Residues to a linear fit relative to a $300 \mathrm{mV}$ output voltage range for the differential configuration.

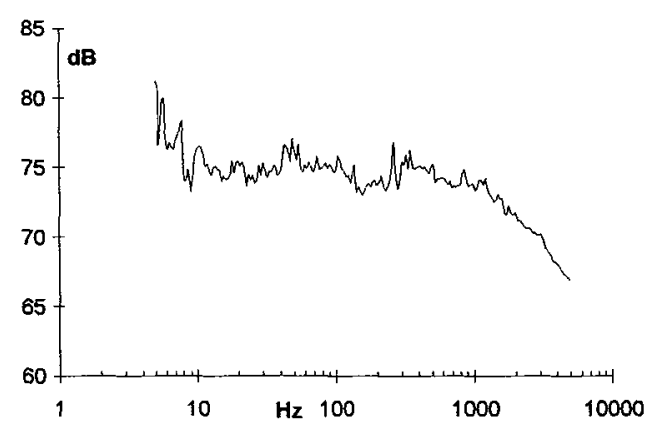

Figure 3: Differential Common Mode Rejection Ratio.

The series noise for the differential configuration at $77 \mathrm{~K}$ and $4.2 \mathrm{~K}$ is shown in Figure 4 and Figure 5 respectively. As can be seen the noise at $4.2 \mathrm{~K}$ is larger than at $77 \mathrm{~K}$, differently from what we expected from a previous characterization of this monolithic process [3]. The dominant noise source has a behavior $A_{f} / f$, with a coefficient $A_{f}$ of about $3.6 \times 10^{-13} V^{2}$ at $77 \mathrm{~K}$, and $6.4 \times 10^{-13} \mathrm{~V}^{2}$ at $4.2 \mathrm{~K}$. Frequency bandwidth is about $2.5 \mathrm{MHz}$. The same consideration regarding the noise applies to the single-ended configuration except for the fact that each channel shows a series noise smaller by a factor $\sqrt{2}$. 


\section{THE BUFFER/LED DRIVER (B/LD)}

The B/LD was designed following the requirements of a Liquid Argon Preshower detector under development at CERN [4]. The temperature of operation of the whole system is $87 \mathrm{~K}$, the LAr temperature. The aim of the circuit is to convert the voltage signal at the output of the preamplifier into a current, suitable to drive a LED. This is located in LAr, and it is used to drive a fiber optic, converting the signal current into an optical signal. The requirements to the $\mathrm{B} / \mathrm{LD}$ are to be fast, as the expected rate of the incoming events will be high, to dissipate very low DC standing power because a large number of

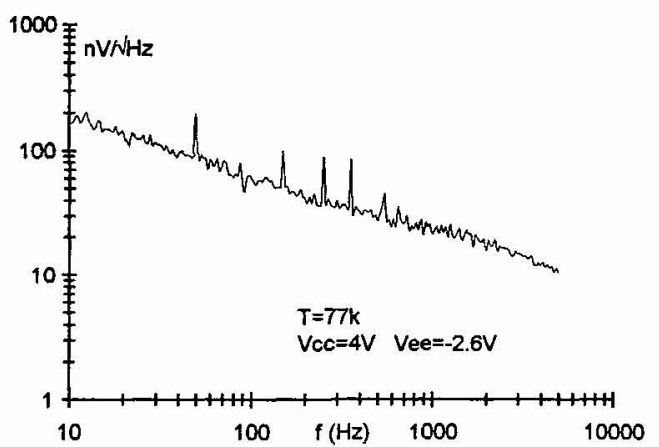

Figure 4: Series noise at $77 \mathrm{~K}$ of the D/SVSP.

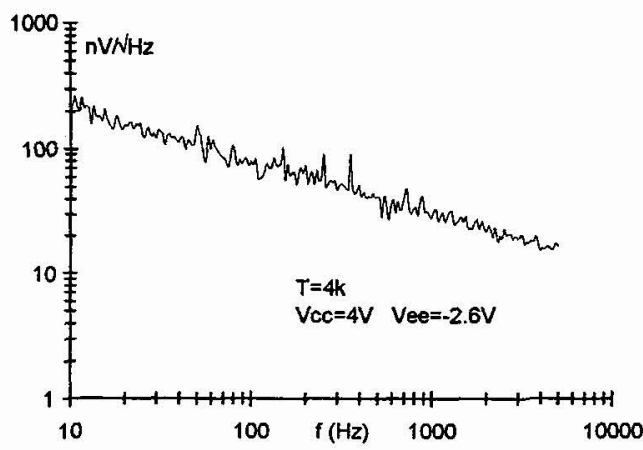

Figure 5: Series noise at $4 \mathrm{~K}$ of the D/SVSP.

channels are foreseen, and to be able to drive a large signal current, up to $100 \mathrm{~mA}$ with an integral nonlinearity of less than $1 \%$.

The monolithic circuit we have realized satisfy all those requirements. Its schematic diagram is shown in Figure 6. The photograph of an output buffered voltage signal of $2 \mathrm{~V}$ is illustrated in Figure 7. As can be

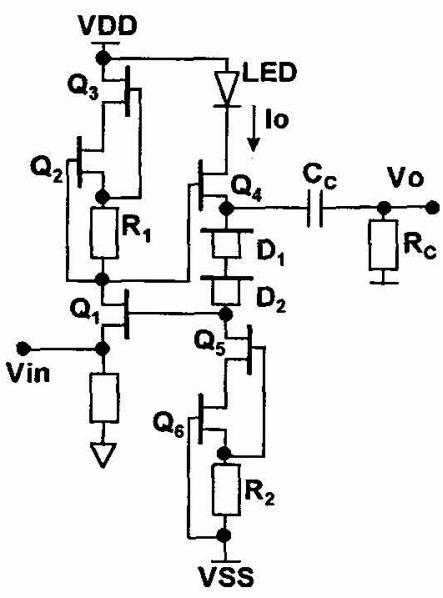

Figure 6: Schematic diagram of the B/LD.

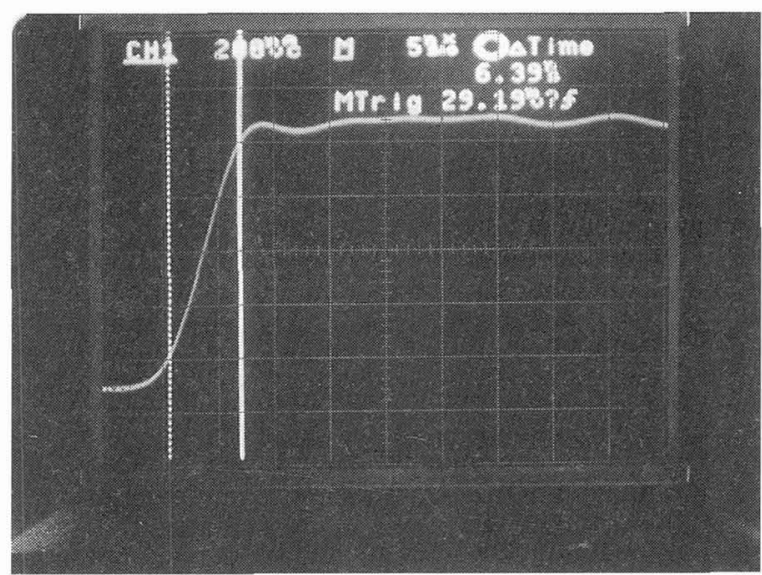

Figure 7: Photograph of a $2 \mathrm{~V}$ output signal voltage at the $\mathrm{V}_{\mathrm{O}}$ output. The signal is taken at the receiving end of a terminated coaxial cable. Vertical Scale: $200 \mathrm{mV} /$ div; horizontal scale:5ns/div.

seen in Figure 6 only a few transistors were used to implement the network. The circuit is a nonsymmetrical differential amplifier having the non-inverting input at the source of $Q_{1}$, while the inverting input is at the gate of $Q_{1}$. Q1 is loaded by a bootstrapped current source $R_{1}, Q_{2}$ and $Q_{3} ; Q_{4}$ is the output buffer transistor. In the configuration shown the network has a unity feedback return ratio (between the 
gate of the input transistor $Q_{1}$ and the output, at the source of the transistor $Q_{4}$, via the two shifting diodes $D_{1}$ and $D_{2}$ ). The circuit can operate as a unity gain buffer, taking the output voltage at $V_{0}$, or as a transconductance amplifier having a gain $\mathrm{g}_{\mathrm{m}}=1 / \mathrm{R}_{\mathrm{C}}$ by reading the output current at the drain of $\mathrm{Q}_{4}$.

We can calculate the closed-loop gain. The open-loop gains for the non-inverting input $\mathrm{A}^{+}$and for the inverting input $\mathrm{A}^{-}$are:

$$
A^{+}=\frac{V_{O}}{V_{Q 1 S}}=\frac{g_{m 4} R_{c}}{1+g_{m 4} R_{C}}\left(1+g_{m 1} R_{D S 1}\right) \quad A^{-}=\frac{V_{O}}{V_{Q 1 G}}=\frac{g_{m 4} R_{c}}{1+g_{m 4} R_{C}} g_{m 1} R_{D S 1}
$$

in eq.(1) $R_{D S 1}$ is the dynamic Drain to Source resistance of $Q_{1}$. The dynamic impedance $R_{\text {GEN }}$ of the bootstrapped current source $R_{1}, Q_{2}$ and $Q_{3}$ was neglected as much greater than $R_{D S 1}$. The gain $A^{+}$is about 25 when the output loading impedance $R_{C}$ is $100 \Omega, 11$ when it is $20 \Omega$. Finally the closed-loop gain from $V_{\text {OUT }}$ to $V_{\text {in }}$ becomes, taking into account that the feedback return ratio, $\beta$, is unity:

$$
A_{C L}=\frac{A^{+}}{1+\beta A^{-}} \approx \frac{1}{1+\frac{1}{g_{m 4} R_{C}\left(1+g_{m 1} R_{D S 1}\right)}}
$$

$A_{C L}$ results larger than 0.9 when the load resistor $R_{C}$ is as low as $20 \Omega$. This is obtained by considering that the term $\mu=g_{m 1} R_{D S 1}$ is about 28 and $g_{m 4} 35 \mathrm{~mA} / \mathrm{V}$ in eq.(2). The feedback action increases the open loop input impedance $\left(R_{\mathrm{GEN}}+\mathbf{R}_{\mathrm{DS} 1}\right) /\left(1+\mathrm{g}_{\mathrm{m} 1} \mathbf{R}_{\mathrm{DS} 1}\right)$ and decrease the output impedance $1 / \mathrm{g}_{\mathrm{m} 4}$ to:

$$
R_{i} \approx \frac{R_{D S 1}+R_{G E N}}{1+g_{m 1} R_{D S 1}}\left(1+\frac{g_{m 4} R_{C}}{1+g_{m 4} R_{C}} g_{m 1} R_{D S 1}\right) \quad R_{\circ} \approx \frac{1}{g_{m 4}} \frac{1}{1+g_{m l} R_{D S 1}} .
$$

$R_{i}$ is estimated slightly less than $500 \mathrm{k} \Omega$, much greater than the impedance of the source, $50 \Omega$. The simulated output impedance $R_{0}$ was about $3 \Omega$, the measured one was $4 \Omega$, included stray effects.

The occupation area of the chip is $1 \times 0.4 \mathrm{~mm}^{2}$, with an input transistor having an area of $2 \times 1000 \mu \mathrm{m}^{2}$. All the characteristics were measured at a temperature of operation of $77 \mathrm{~K}$. The positive supply voltage was set to $5.5 \mathrm{~V}$, the negative one to $-2.5 \mathrm{~V}$. The total power dissipation was $12.6 \mathrm{~mW}$.

The $\mathrm{B} / \mathrm{LD}$ was configured for three different operations. Figure 7 shows that the circuit is able to work as a unity gain buffer, even with large signals. Very good results were obtained when the network was operated as a transconductance amplifier. Figure 8 shows the integral non-linearity of the output signal current. The current was developed across a $2.5 \Omega$ resistor connected between the drain of $\mathrm{Q}_{4}$ and the positive supply voltage $V_{D D}$. The signal was filtered by a RC-CR shaper (one integrator and one differentiator) having $20 \mathrm{~ns}$ peaking time. As can be seen the error is less than $1 \%$ referred to a range of $100 \mathrm{~mA}$. The rise time even for the large signal was less then $9 \mathrm{~ns}$, as illustrated in Figure 9.

With $\mathrm{B} / \mathrm{LD}$ was also possible to realize a $\mathrm{RC}-\mathrm{CR}$ shaping filter of $50 \mathrm{~ns}$ peaking time. The implementation was realized as follows. Resistor $R_{C}$ and capacitor $C_{C}$ were set $20 \Omega$ and $2.5 \mathrm{nF}$ respectively, acting as differentiator. A $50 \Omega$ resistor was connected between the drain and the supply voltage of $\mathrm{Q}_{4}$. The receiving end of a coaxial cable was $A C$ coupled to the $\mathrm{Q}_{4}$ drain with an integrator formed with $50 \Omega$ shunted with $2 \mathrm{nF}$. This way the line resulted terminated and the low-pass filter was effective in filtering the electromagnetic interferences acting on the cable. No distortions or reflections were observed in the output pulse also for $10 \mathrm{~m}$ of cable length.

The series noise, measured in a $50 \mathrm{MHz}$ frequency range, had a $1 / f$ component with $A_{f} \approx 10^{-11} V^{2}$ and a white component of slightly less then $1.5 \mathrm{nV} / \mathrm{JHz}$, resulting in about $4.5 \mathrm{MHz}$ corner frequency. 


\section{CONCLUSIONS}

Two new linear ASIC's for cryogenic use have been realized using GaAs technology. A voltage preamplifier able to work as a two channel single-ended input preamplifier or as a differential preamplifier was able to work down to $4.2 \mathrm{~K}$. Power dissipation can range between $20 \mathrm{~mW}$ and $37 \mathrm{~mW}$, depending on dynamic and noise requirements. At $4.2 \mathrm{~K}$ in the $100 \mathrm{kHz}$ frequency range the noise was $1 / \mathrm{f}$ dominant with a power spectrum at $1 \mathrm{~Hz}$ of $6.4 \times 10^{-13} \mathrm{~V}^{2} / \mathrm{Hz}$. A fast unity-gain voltage buffer and transconductance amplifier was realized to operate at $87 \mathrm{~K}$. It is able to amplify large signals and to drive a $100 \mathrm{~mA}$ current into a LED, for analog optical signal transmission from a cryogenic environment. Power dissipation is 12.6 $\mathrm{mW}$, the rise time of a signal of $100 \mathrm{~mA}$ on $2.5 \Omega$ was $8 \mathrm{~ns}$. The input series noise has a $1 / \mathrm{f}$ component having an intensity $A_{f}$ of about $10^{-11} \mathrm{~V}^{2}$, followed by a white noise term slightly less than $1.5 \mathrm{nV} / \sqrt{\mathrm{Hz}}$. The corner frequency is about $4.5 \mathrm{MHz}$.

The voltage-sensitive preamplifier was designed to read-out the signal of bolometric detectors, while the Buffer/Led Driver was intended to amplify signals coming from a Liquid Argon Preshower detector at CERN.

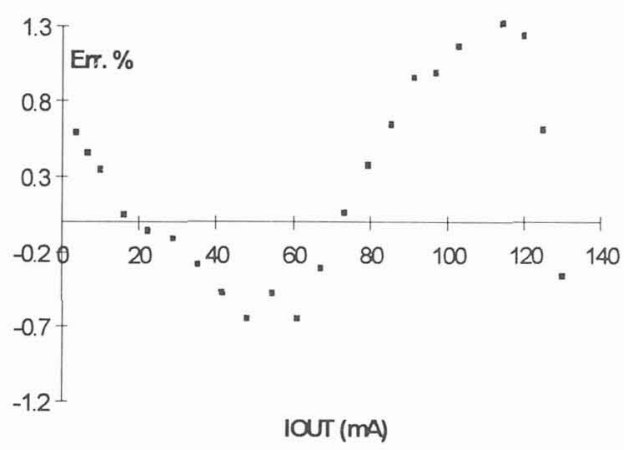

Figure 8: Integral non linearity for the output current of the $\mathrm{B} / \mathrm{LD}$

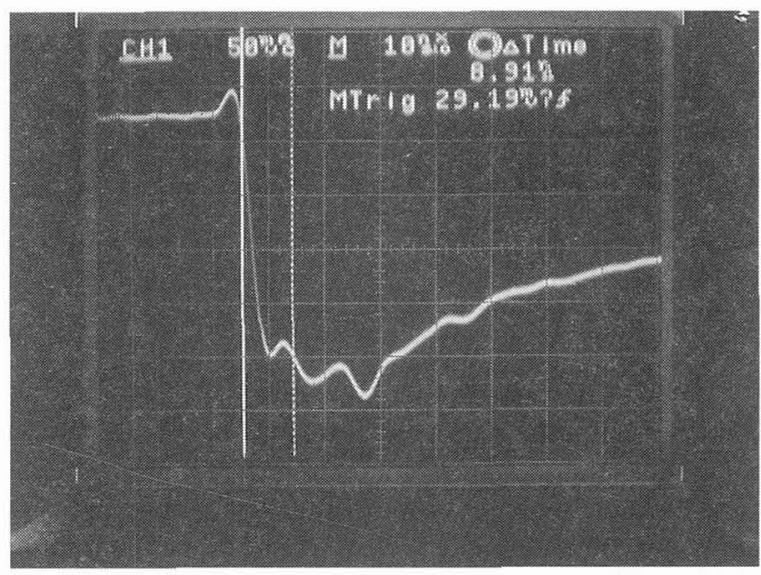

Figure 9: Photograph of a $100 \mathrm{~mA}$ output current signal on $2.5 \Omega$ drain load resistor.

Vertical Scale: $50 \mathrm{mV} / \mathrm{div}$; horizontal scale: $10 \mathrm{~ns} / \mathrm{div}$.

\section{ACKNOWLEDGEMENTS}

The technical support of Maurizio Perego and the help given by our student Giampaolo Sablich in taking the measurements are much appreciated.

\section{REFERENCES}

[1] See for Instance Journal of Low Temperature Physics, 93 (1993), pp.185-858, Special Issue of 'The $\mathrm{V}^{\mathrm{TH}}$ Int. Works. On Low Temperature Detectors'.

[2] A.Alessandrello et al., Nucl. Instr. And Meth. In Phys. Res., A289 (1990), 426-437.

[3] D.V.Camin, G.Pessina and E.Previtali, IEEE Tran. On Nucl. Sci., 41 (1994), 1260-1266.

[4] B.Aubert et al., Nucl. Instr. And Meth. In Phys. Res., A330 (1993), 405-415. 